\title{
Temporomandibular joint disorders: a case-based review on the presentation and conservative management of the condition Dearbhla McEleney; Shadaba Ahmed, FRCS
}

\section{Dearbhla is a fourth-year student at Lancaster Medical School.}

\section{INTRODUCTION}

In 1934, the otolaryngologist Dr James Costen first recognised symptoms arising from the temporomandibular joint (TMJ).(I) Pain in the joint and surrounding muscles, as a result of occlusal disturbance, were the initial characteristics for what was known as 'Costen's Syndrome'.(1) Costen's Syndrome was criticised for its poor diagnostic accuracy until further developments arose in the 1980s, recognising the multifactorial nature of the condition. (I) The American Academy of Orofacial Pain revolutionised the definition of TMJ disorders as 'a collective term embracing a number of clinical problems that involve the masticatory muscles, the TMJ and associated structures, or both'. (2) This disorder is one of the most common orofacial pain complaints, with $6-12 \%$ of the population aged between 20 and 40 years, presenting to general practitioners or dentists, with subsequent referrals to maxillofacial specialists. ${ }^{(3)}$ These figures represent the increasing demand on secondary healthcare resources, thus emphasising the need for early diagnosis and subsequent conservative management.

A TMJ disorder refers to problems involving the masticatory muscles, the TMJ and the surrounding bony and soft tissue. ${ }^{(3)}$ The aetiology of this condition is multifactorial, yet, the pathophysiology is ill-understood. ${ }^{(4)}$ TMJ disorders are mistakenly classified as a single disorder, when in fact they can be classified into two groups with further sub-diagnoses: articular disorders; and non-articular disorders. ${ }^{(3,5)}$ Fifty percent of cases are diagnosed with myofascial pain, a non-articular disorder resulting from pain in the musculature with or without limited opening of the joint. ${ }^{(3)}$ Other non-articular causes include: muscle spasms; acute muscle strain; and chronic pain conditions such as fibromyalgia. ${ }^{(3)}$ The articular disorders include: direct trauma; osteoarthritis; infectious; psoriatic; and rheumatoid arthritis. ${ }^{(3)}$

Currently, 33\% of the population experience at least one symptom related to TMJ disorders. ${ }^{(5)}$ These symptoms differ from person to person and thus can have a variety of detrimental impacts to ones quality of life: $6-12 \%$ of this population seek medical attention. ${ }^{(6)}$ Failure to recognise psychological factors contributing to the physical symptoms associated with this disorder has resulted in misdiagnosis and unnecessary patient referrals. (7) The management of TMJ disorders has evolved; what was once a condition managed by general practitioners is now one managed by maxillofacial specialists or dentists. ${ }^{\left({ }^{8}\right)}$

The majority (80\%) of patients referred to specialists only require conservative treatment, suggesting that this condition could be managed in primary care. ${ }^{(8)}$ With this is mind, this is a case-based review exploring the presentation, diagnosis, and conservative management of the condition. Particular emphasis will be given to the potential to minimise patient referrals and the burden on secondary healthcare resources.

\section{METHOD}

The selection of cases was undertaken by consultants from ENT and maxillofacial departments. The two consultants kept record of any patient who presented between November 2013 and February 2014 with TMJ symptoms and who were given a diagnosis of a TMJ disorder or subgroup within this. Two cases are singled out for detailed discussion to illustrate modern management.

\begin{tabular}{|c|c|c|c|c|}
\hline Symptom & Case I & Case 2 & Case 3 & Case 4 \\
\hline Pain in the TMJ area & Yes - bilateral & Yes - left-sided facial pain & Yes - bilateral & Yes - pain in the right TMJ \\
\hline $\begin{array}{l}\text { Tenderness of muscles } \\
\text { radiating from TMJ to } \\
\text { maxilla/neck/shoulders }\end{array}$ & $\begin{array}{l}\text { Yes - bilaterally spreads } \\
\text { forward across her } \\
\text { zygomasup into her } \\
\text { temporal region }\end{array}$ & No & $\begin{array}{l}\text { Yes - bilaterally } \\
\text { accompanied by muscle } \\
\text { spasm }\end{array}$ & $\begin{array}{l}\text { Yes - tenderness in } \\
\text { the right TMJ }\end{array}$ \\
\hline Headache/migraine & No & No & No & No \\
\hline Otalgia +/- tinnitus & $\begin{array}{l}\text { Previous history of pain } \\
\text { in both ears but has } \\
\text { resolved }\end{array}$ & No & $\begin{array}{l}\text { History of pain in right } \\
\text { ear }\end{array}$ & No \\
\hline Joint noise & No & Yes - clicking in both TMJs & No & $\begin{array}{l}\text { Yes - clicking on right side } \\
\text { with opening }\end{array}$ \\
\hline $\begin{array}{l}\text { Limited movement } \\
\text { including locking of joint }\end{array}$ & No & $\begin{array}{l}\text { Past history of limited } \\
\text { opening }\end{array}$ & $\begin{array}{l}\text { Yes - limited movement } \\
\text { as a result of reluctance } \\
\text { due to pain }\end{array}$ & No \\
\hline
\end{tabular}




\section{RESULTS}

Four cases in total were identified in the three-month period. They were all female with ages between 32 and 56. Presenting symptoms are summarised in table I. The two cases selected for discussion were diagnosed with different subgroups ofTMJ disorders, whereas the other two cases had the same diagnosis.

\section{DISCUSSION}

\section{Anatomy and physiology of TMJ}

The TMJ is a synovial joint located between the condylar process of the mandible, the articular fossa and the articular eminence of the temporal bone. The fibrocartilage articular disc is attached to the lateral pterygoid muscles anteriorly and the joint capsule. This separates the joint into upper and lower cavities. The articular disc, with a peripheral blood supply and an avascular centre, has numerous functions, including stabilisation of the condyle, the ability to adapt to maintain its relationship with the articular surface of the condyle during mandibular movements, and the production of fluid to provide lubrication, thereby decreasing friction on the condyle. The synovial fluid functions as a transportation medium for nutrients and waste products to areas devoid of vasculature. The posterior attachment of the articular disc to the retrodiscal tissue, an area of loose, highly vascularised and innervated connective tissue, is of great clinical significance. ${ }^{(1)}$ If the disc is displaced, then the condyle will articulate with the posterior retrodiscal lamina, causing significant pain. ${ }^{(1)}$

The majority of TMJ movements rely on a group of muscles, collectively known as the masticatory muscles. Their primary role is to close the jaw; however, with the aid of gravity they are also involved in opening it. The masseter, a masticatory muscle, originates at the lower border and deep surface of the zygomatic arch inserting into the lateral surface of the ramus of the mandible. This muscle is involved in the elevation and protrusion of the mandible.

\section{The presentation of TMJ disorders}

The recognised signs and symptoms related to TMJ disorders have changed since Costen's initial findings in 1934 and are variable between patients (see table I).(I) Muscular tenderness may occur in the face involving the masseter, temporalis, medial or lateral pterygoid muscles, and in some cases, the neck and shoulder muscles. ${ }^{(I)}$ Pain in the TMJ area which may radiate to the face, towards the maxilla and further down to the neck, is yet another symptom that may be experienced.( ${ }^{(9)}$

'Joint noise' whilst moving the TMJ (eg clicking or cracking) has also been reported. ${ }^{(4)}$ This noise can be accompanied by locking, limitation or deviation in joint opening.(1) These symptoms may be accompanied by headache, tinnitus, or reduced hearing.(9)

Headaches of variable severity can occur and are usually first thing in the morning. ${ }^{(10)}$ Much debate surrounds whether TMJ disorders are a risk factor for the development of headaches and whether those suffering with myofascial pain are prone to tension-type headaches. ${ }^{(10)}$ An increasing number of cases are presenting to ENT clinics with otalgia, a common symptom, often overlooked in the diagnosis ofTMJ disorders. ${ }^{(1)}$
In some cases, the symptoms may be acute and self-limiting. ${ }^{(9)}$ However, this condition may be chronic and debilitating, having similar consequences on a person's life as any other chronic pain syndrome, eg fibromyalgia. ${ }^{(9)}$ This sometimes results in multiple referrals to different specialties for a condition whose features are more in keeping with a generalised pain disorder that is better managed as such.

\section{Aetiology and risk factors of TMJ disorders}

In patients aged 18-45 years, TMJ disorders are twice as prevalent in females. ${ }^{(12)}$ As part of a comprehensive patient history, it is essential to explore risk factors that could contribute to TMJ dysfunction. Initiating factors such as trauma (eg during sports), recent orthodontic or dental treatment, in particular third molar removal, may contribute to the onset of symptoms. ${ }^{(13)}$ Debate surrounds whether TMJ disorders are a consequence of poor dental occlusion, as evidence shows that occlusal treatment has little benefit. ${ }^{(14)}$

Predisposing factors such as nail biting, chewing a pen, and holding a telephone between the shoulder and jaw may increase the risk of developing symptoms. ${ }^{(13)}$ Nail biting and other habits may be symptoms of stress and anxiety; therefore, it is valuable to pick up such cues as part of a general enquiry as to levels of stress to explore if the patient is under any stress (eg financial/emotional). ${ }^{(15)}$ Other habits, more obviously related to the joint itself, such as daytime clenching, grinding of the teeth, and nocturnal bruxism, can contribute to dysfunction and pain. ${ }^{(13)}$ The link between these habits and stress is recognised. Paradoxically, according to some reports, the medications used to successfully treat these conditions may result in the development, or worsening, of bruxism..$^{(16)}$

It is clear that in presenting cases it may be difficult to distinguish the aetiology of an individual's symptoms, as a multitude of factors are associated with TMJ disorders. Often in many cases, no obvious cause is found resulting in difficulty with patient management. ${ }^{(4,16)}$ Doctors working in the acute secondary care sector may not have the skills or inclination, or indeed time, to enquire of and manage the psychological distress. Therefore, a primary care setting, where a strong doctor-patient relationship exists, would be preferable to recognise these features and address any concerns or expectations that the patient may have.

\section{Diagnostic criteria}

History should explore the aforementioned signs, symptoms and risk factors of the condition prior to examination. In 1992 , the Research Diagnostic Criteria for Temporomandibular Disorders (RDC/TMD) was introduced as a diagnostic classification tool and has since been accepted as the gold standard. ${ }^{(17)}$ However, its use in a clinical setting can be time consuming, prompting the publication of a modified RDC/TMD called the Clinical Examination Protocol for Temporomandibular Disorders (CEP-TMD). ${ }^{(17)}$

A summary of the diagnostic groups, as described in the RDC/TMD is shown in table 2.

In 2014, a shortened version of the RDC/TMD was produced and renamed the Diagnostic Criteria for Temporomandibular Disorders (DC/TMD). ${ }^{(18)}$ This questionnaire has two 'axes'. Axis I relates to the demographics and biomedical diagnosis and contains a symptoms and demographics questionnaire for the patient and an examination form for the clinician. ${ }^{(18)}$ Axis II 


\begin{tabular}{|l|l|}
\hline Symptoms/signs & Diagnosis \\
\hline $\begin{array}{l}\text { Muscle disorders } \\
\text { Muscle pain +/- limited } \\
\text { movement }\end{array}$ & $\begin{array}{l}\text { Myofascial pain +/- limited } \\
\text { opening }\end{array}$ \\
\hline $\begin{array}{l}\text { Disc displacements } \\
\text { Reproducible clicking } \\
\text { Limited opening with no } \\
\text { clicking } \\
\text { Limited opening with prior } \\
\text { proof of disc displacement }\end{array}$ & $\begin{array}{l}\text { Disc displacement with reduction } \\
\text { Disc displacement without } \\
\text { reduction with limited opening } \\
\text { Disc displacement without } \\
\text { reduction without limited } \\
\text { opening }\end{array}$ \\
\hline $\begin{array}{l}\text { Other joint problems } \\
\text { Crepitus } \\
\text { Joint pain } \\
\text { Crepitus and joint pain }\end{array}$ & $\begin{array}{l}\text { Osteoarthrosis } \\
\text { Arthralgia } \\
\text { Osteoarthritis }\end{array}$ \\
\hline \multicolumn{2}{|c|}{ Table 2 Axis I: biomedical diagnosis (17) } \\
\hline
\end{tabular}

relates to the biopsychosocial aspects and contains tools that further assess the patient and their condition at that point in time (see table 3). ${ }^{(18)}$ The DC/TMD includes concise specifications for examinations: diagnostic trees and tables summarising each sub group; and the criteria that must be met to give each of the diagnoses. ${ }^{(18)}$ Each patient is unique, therefore it is important to recognise that following these guidelines and a 'one approach for all patients', will not always be appropriate. ${ }^{(19)}$ However, the benefit of the DC/TMD will enable all clinicians to follow a step-wise approach to a structured examination protocol, in turn keeping the patients notes up-to-date and enhancing the continued assessment of disease progression. Furthermore, this will aid handover when taking on a multidisciplinary team approach (eg CBT). ${ }^{(19)}$

\begin{tabular}{|l|l|}
\hline Test/questionnaire & What does the questionnaire entail? \\
\hline Pain drawing questionnaire & $\begin{array}{l}\text { Shade different areas of the body, face } \\
\text { or mouth to indicate where pain is } \\
\text { experienced }\end{array}$ \\
\hline $\begin{array}{l}\text { Graded chronic pain scale, } \\
\text { version 2 }\end{array}$ & $\begin{array}{l}\text { Questionnaire detailing the severity } \\
\text { and duration and impact of the pain }\end{array}$ \\
\hline $\begin{array}{l}\text { Jaw functional limitation } \\
\text { scale }\end{array}$ & $\begin{array}{l}\text { A number of questions whereby the } \\
\text { patient must indicate on a scale of } \\
\text { I- I0 the level of jaw limitation over } \\
\text { the past month whilst carrying out } \\
\text { certain activities }\end{array}$ \\
\hline Patient health questionnaire & $\begin{array}{l}\text { Four questions exploring if the patient } \\
\text { has been suffering from symptoms } \\
\text { related to anxiety or depression }\end{array}$ \\
\hline Oral behaviour checklist & $\begin{array}{l}\text { A questionnaire based on the activities } \\
\text { the patient does whilst asleep and } \\
\text { awake during the last month }\end{array}$ \\
\hline \multicolumn{2}{|c|}{ Table 3 Axis II: functional and biopsychosocial assessment (18) } \\
\hline
\end{tabular}

Although said to be a shortened version, the DC/TMD, in particular Axis II, still appears to be lengthy and it is debatable whether or not this could be used in a primary care setting. Following an initial consultation in primary care, completion of the questionnaires at home would alleviate time restraints and allow for the primary care clinician to take more prompt action. This, however, may be unsuccessful as sending a patient home to fill out a questionnaire could still prolong the time for a diagnosis and may still result in patient distress.

\section{Diagnostic imaging}

Although sometimes performed, plain radiographs are not indicated in the diagnosis of TMJ disorders as they rarely contribute to the patient's treatment plan.(1) Advanced imaging, such as computerised tomography (CT) scans, is indicated for patients presenting with pathology that mimics TMJ disorders (eg malignancy of head/neck), but must be justified by the clinician. ${ }^{(1)}$

\section{CASE-BASED DISCUSSION}

Surgical management, such as arthroscopy and arthrocentesis, is required for only a small proportion of patients with the diagnosis ofTMJ disorder. Recent evidence suggests that patients in all branches of medicine benefit from the use of 'patient decision aids', these are online sources that provide information on the treatment options available. ${ }^{(20)}$ The aid specific for TMJ disorders focuses on whether or not individuals should opt for a surgical intervention to alleviate their jaw pain. ${ }^{(21)}$ A previously paternalistic approach to consultations has become patient centred, having a positive impact on the doctor-patient relationship and the overall care of the patient. (22)

\section{Case I: atypical facial pain}

Referred to the maxillofacial department with a one-year history of bilateral facial pain. Her initial presenting complaint of pain in front of both ears was treated as an ear infection and had fluid drained from her ear by the ENT department. She had a few months of relief but re-presented to the maxillofacial department after the pain reoccurred. The pain started in the evenings in front of the right and left ears over the TMJ region and spreads across her zygomas, into her temporal region and across her cheeks bilaterally. On examination, there was no clicking or locking and no pain was noted in the TMJs. Intra-orally, the examination revealed no evidence of grinding but tenderness on palpation of the pterygoid muscles bilaterally. X-ray of the joints did not reveal any abnormalities. The pain eased with ibuprofen but the persistence had been causing the patient distress. This patient had a history of depression and was taking 10mg amitriptyline daily. Throughout the consultation the patient was upset and admitted to a particularly stressful work life.

\section{Case 2:TMJ pain dysfunction}

Referred from ENT to the maxillofacial department regarding TMJ pain. She presented with a long history of clicking from the TMJs and recently experienced pain on the left side of her face in the region of the TMJ. She described the pain being worse at night. The patient had a past history of joint stiffness with limited opening of the jaw, particularly in the morning, but denies bruxism. Extra-orally, the examination revealed mild clicking from both TMJs but good mouth opening and intraorally, no evidence of teeth grinding.

\section{Conservative management options I. Patient reassurance and information sheet, including a home exercise programme}

The management plan for both cases involved discharging the patients with an information sheet, following diagnosis and reassurance from the consultant. A 2010 study showed that patients who repeatedly present to primary care with the aforementioned symptoms, without receiving a diagnosis, risk suffering from anxiety, consequently exacerbating their condition. $^{(7)}$ 
It was also found that those patients without a diagnosis questioned their symptoms and believed that others, such as clinicians and family members, doubted the legitimacy of their complaints. ${ }^{(7)}$ Therefore, it is essential that the patient is diagnosed correctly and reassured in their consultation in order to help them understand and cope with their condition.

The patient information sheet provided for these two cases by the Morecambe Bay Maxillofacial Services, educates the patient regarding TMJ disorders, their symptoms and associated aggravating factors (see appendix). Furthermore, possible solutions to the underlying problems are provided, such as relaxation therapies for patients to control stress, thus reducing parafunctional habits such as nail biting. ${ }^{(4)}$ A step-by-step guide on jaw exercises is provided to prevent clicking of the jaw and focuses on correcting muscle imbalance. This strengthens the muscles, which in turn enables them to relax, thereby, relieving pain and tenderness. ${ }^{(23)}$

In acute TMJ disorder attacks, however, exercises are not recommended as they can further damage the joint and exacerbate symptoms. ${ }^{(19)}$ In these cases, resting the joint, following a soft-food diet, and avoiding opening the mouth too wide, are recommended. ${ }^{(19)}$ As with any other joint pain, heat or ice applied to the joint may also provide relief. ${ }^{(4)}$ Finally, it is essential to inform the patient that in most cases TMJ disorders are not sinister and usually respond to the conservative measures, but may return after several years. ${ }^{(4)}$

\section{Medication}

The evidence basis for the current practice of medication usage is summarised below.

\section{Medication}

As a first-line medication for TMJ disorders, patients are offered NSAIDs. According to a British Medical Journal meta-analysis, topical NSAIDs were superior to placebo in the first two weeks of treatment for superficial joint pain. ${ }^{(24)}$

A Cochrane review assessing the effectiveness of pharmacological interventions for pain in patients with TMJ disorders compared the use of a variety of oral and topical NSAIDs against placebos. ${ }^{(25)}$

\section{Cochrane Database Systematic Review ${ }^{(25)}$}

One study by Ta and Dionne, proved significant pain reduction with naproxen in comparison to placebo over a six-week period (mean difference -1.70; 95\% Cl-2.14 to - I.25; P <0.00I).

Furthermore, Lobo et al's study showed TMJ pain reduction with topical methyl salicylate in comparison to placebo (mean difference $-0.90 ; 95 \% \mathrm{Cl}-1.35$ to $-0.45 ; \mathrm{P}<0.00 \mathrm{I}$ ). Whereas, Roldan et al's study comparing piroxicam with a placebo over 15 days showed no statistical significance (risk ratio 0.7I; $95 \% \mathrm{CI} 0.29$ to $1.76 ; P=0.46$ ).

Anti-convulsants such as Gabapentin, and benzodiazepines such as Clonazepam were also reviewed. Kimos et al's study showed significant pain reduction over placebo with the use of Gabapentin for those with TMJ pain (mean difference -3.20; $95 \% \mathrm{Cl}-4.7 \mathrm{I}$ to I.69; P < 0.00 I). Yet, Harkins et al's study showed no statistical difference in pain management between Clonazepam and placebo (mean difference $-0.74 ; 95 \% \mathrm{Cl}-3.14$ to $1.66 ; \mathrm{P}=0.55$ ).

Therefore, the Cochrane review remains inconclusive, as insufficient evidence exists to accept or reject the aforementioned drugs as an adequate treatment option, highlighting the need for further RCT's and meta-analyses in the pharmacological management of TMJ disorders.

\section{Cognitive behavioural therapy (CBT)}

With no physical abnormalities and a history of depression, a diagnosis of atypical facial pain was given to the patient in case I. CBT was considered as a potential management option.
CBT involves helping the patient change how they think and behave, whilst recognising the physical outcomes that emotions may produce.(26) This treatment focuses on preventing parafunctional habits and reducing clenching or bruxing. ${ }^{(27)}$ It also encourages patients with 'catastrophising cognitions' to change their perception of the condition, from an intolerable one to a manageable one, thereby reducing distress. ${ }^{(27)} \mathrm{CBT}$ has proven to be effective for these patients in particular, although, weak evidence suggests that it is beneficial for symptomatic relief of those without catastrophising cognitions. ${ }^{(27,28)}$

CBT alone may not directly improve pain; however, it improves sleep, thus, improving ones quality of life. (29) A combined treatment of amitriptyline and CBT has shown to be effective in the treatment ofTMJ pain and should be considered as a treatment option for patients. ${ }^{(29)}$ Research has also shown that self-management techniques combined with the use of NSAIDs is extremely effective, whereas, further research is necessary to establish if incorporating CBT could enhance this combination therapy. ${ }^{(27)}$

\section{Intra-oral appliances}

On examination of the two aforementioned patients, no evidence of bruxism was present. Some patients do, however, show signs of bruxism and are advised to consider the use of a bite splint to prevent this. ${ }^{(8)}$ Many types of splints exist, yet evidence suggests that no single splint is superior to another and a simple lower soft splint is adequate. ${ }^{(8)}$ Furthermore, this enables the masseter muscles to relax, reducing pain and tenderness. ${ }^{(30)}$ Although they can be beneficial, splints may result in further problems through the alteration of the patient's occlusion. ${ }^{(30)}$

\section{CONCLUSION}

Knowledge and understanding regarding TMJ disorders has evolved, yet many patients are still being stepped up from primary care without a diagnosis or appropriate management.

With the publication of the DC/TMD, there is hope that the uptake and awareness of this increases in an attempt to screen, evaluate and diagnose patients promptly. However, attempts to promote the DC/TMD may prove to be futile as this is still as impractical as its predecessor.

Currently, it is difficult to tell if the CEP-TMD will be a superior tool when compared to the DC/TMD. As with the DC/TMD, only time will tell as to whether or not the CEP-TMD will be suitable in effectively diagnosing patients.

It could be suggested that by conducting future studies and directly comparing the DC/TMD with the CEP-TMD, statistically significant evidence will become available to demonstrate that one is more useful than the other.

Overall, the future successful management ofTMJ disorders relies on its prompt and confident diagnosis by primary care clinicians. This could be improved by increasing GP and dental awareness of the DC/TMD and CEP-TMD, along with education regarding the use of conservative management techniques. The role of the acute hospital sector, while important for excluding serious conditions and offering surgery to a selected few patients, is limited. The role of the patient in overcoming the helplessness that results from a series of secondary care appointments with consequent overlapping diagnoses cannot be overstated. 


\section{Commentary...}

\section{Tim Norton is senior lecturer in Cognitive Behavioural Therapy at the University of Cumbria.}

Your accompanying paper describes four patients with temporomandibular joint disorders. Common to all of them is the experience of TMJ pain. The author recommends exploring symptoms of stress and anxiety. This is to be encouraged; however, the experience of pain itself may lead to the circularity of symptoms whereby the very experience of pain is sufficient to link with insomnia, poor information processing, avoidance, disruption to activities of daily living and possible consequences of anxiety and depression.

Although pain is a personal and subjective experience, the fact that someone is experiencing pain is often apparent to others. People who have pain may vocalise their distress by moaning, crying or complaining. They may exhibit painrelated body postures, facial expressions or grimacing that communicate the fact that pain is being experienced. Resting in bed, taking medication and moving in a guarded fashion can all be indications of pain-related behaviour. Increasingly, these are viewed as an important target in pain assessment. These verbal and non-verbal behaviours have been called pain behaviours because they serve to communicate the fact that pain is being experienced. ${ }^{(1)}$

Over the past three decades, cognitive-behavioural therapy (CBT) has become a first-line psychosocial treatment for individuals with chronic pain. Evidence for efficacy in improving pain and pain-related problems across a wide spectrum of chronic pain syndromes has come from multiple randomised controlled trials. ${ }^{(2)}$ CBT has been tailored to, and found beneficial for, special populations with chronic pain, including children and older adults. ${ }^{(2)}$

Persistent pain and persistent insomnia commonly occur together. ${ }^{(3,4)}$ Pain and sleep disturbance similarly often cooccur, but as the correlation between actual severity of pain and insomnia is weak it is suggested that, rather than causing one another, they share a common cause. ${ }^{(5)}$ Insomnia correlates strongly to emotionally-laden or affective pain. Patients are asked to describe their pain against the emotional descriptors such as 'exhausting', 'sickening', 'fearful' and 'punishing'. As a result of poor sleep, daytime fatigue and poor processing of information become common factors amongst pain sufferers. Intolerance to, and preoccupation with, pain develops. In turn this may lead to behavioural avoidance, increased time to ruminate on personal problems and the danger of lapsing into depressed mood and increased perception of pain.

The biopsychosocial model of pain is concerned with not only illness, but also physical discomfort, emotional distress and behavioural limitations associated with pain and disability. ${ }^{(6)}$ CBT attempts to offer help with the perception of pain and the behavioural and emotional consequences. Your author outlines the model of CBT for pain, competently, though perhaps underestimating the importance of such interventions. There is general consensus that combined biomedical and biopsychosocial methods best support the assessment and management of the cardinal features of TMJ pain, ie pain and dysfunction or physical (peripheral) and psychosocial (central) factors. ${ }^{(7)}$

CBT interventions cover many different techniques. Fundamental to these are changes in behaviours and cognitions to bring about shifts in mood. Mood disturbance is common in patients suffering with pain and it is this that CBT aims to work with. Achieving change in mood and daytime fatigue through CBT for insomnia can lead to early gains in therapy. ${ }^{(8)}$ Principal approaches to CBT include: behavioural experiments to challenge and disconfirm faulty thinking and beliefs; cognitive restructuring to question and guide discovery of more appropriate, helpful ways of thinking; and behavioural activation to challenge inactivity. ${ }^{(9)}$ This should be seen as intensive psychological therapy, carried out by skilled practitioners, ideally attached to pain clinics and working alongside the pain management team, but further development of the workforce and acceptance of these ideas in primary care settings may mean that fewer patients will need to visit hospital to get effective treatment.

\section{REFERENCES}

I. Keefe FJ, Smith, S. The assessment of pain behaviour: implications for applied psychophysiology and future research directions. Appl Psychophysiol Biofeedback 2002;27:2

2. Ehde DM, Dillworth TM,Turner J. Cognitive-behavioral therapy for individuals with chronic pain: efficacy, innovations, and directions for research. Am Psychol 20। 4;69(2): 153-66 (Special issue: Chronic Pain and Psychology)

3. National Institutes of Health. National Institutes of Health State of the Science Conference: statement on manifestations and management of chronic insomnia in adults. Sleep 2005;28(9): 1049-57

4. Roth T. Insomnia: definition, prevalence. Etiology, and consequences. J Clin Sleep Med 2007;3(5 Suppl):S7- I0

5. Tang NKY, Wright KJ, Salkovskis PM. Prevalence and correlates of clinical insomnia co-occurring with chronic back pain. J Sleep Res 2007; 16:85-95

6. Grant M. Pain control with EMDR. Kew, Australia: Grant; 2012

7. Suvinen TI, Reade PC, Kemppainen P, Kononen M, Dworkin SF. Review of aetiological concepts of temporomandibular pain disorders: towards a biopsychosocial model for integration of physical disorder factors with psychological and psychosocial illness impact factors. Eur J Pain 2005;9(6):6 I3-33

8. Harvey AG. A cognitive model of insomnia. Behav Res Ther 2002:40(8):869-93

9. Westbrook D, Kennerley H, Kirk J. An introduction to cognitive behaviour therapy skills and applications. 2nd Ed. London: Sage; 201 I 


\section{REFERENCES}

I. Durham J. Oral surgery: part 3. Temporomandibular disorders. Br Dent J 2013;215(7):331-7

2. American Academy of Orofacial Pain. Orofacial pain : guidelines for assessment, diagnosis, and management. Chicago: Quintessence; 1996

3. Liu F, Steinkeler A. Epidemiology, diagnosis, and treatment of temporomandibular disorders. Dent Clin North Am 20। 3;57(3):465-79

4. British Association of Oral and Maxillofacial Surgeons. Jaw Joint Problems. Available from:

http://www.baoms.org.uk/What_is_Oral_and_Maxillofacial_ Surgery/Sub_specialist_Areas/Jaw_Joint_Problems (Accessed 12 April 2014)

5. Wright EF, North SL. Management and treatment of temporomandibular disorders: a clinical perspective. J Man Manip Ther 2009; I 7(4):247-54

6. de Freitas RF, Ferreira MA, Barbosa GA, Calderon PS. Counselling and self-management therapies for temporomandibular disorders: a systematic review. J Oral Rehabil 20 |3;40( | I):864-74

7. Durham J, Steele JG, Wassell RW, Exley C. Living with uncertainty: temporomandibular disorders. J Dent Res 2010;89(8):827-30

8. Sidebottom AJ. Current thinking in temporomandibular joint management. Br J Oral Maxillofac Surg 2009;47(2):91-4

9. Scrivani SJ, Keith DA, Kaban LB. Temporomandibular disorders. N Engl J Med 2008;359(25):2693-705

10. Karibe H, Goddard G, Okubo M. Comparison of masticatory muscle myofascial pain in patients with and without a chief complaint of headache. Cranio 2014:32(I):57-62

I I. Ely JW, Hansen MR, Clark EC. Diagnosis of ear pain. Am Fam Physician 2008;77(5):62 I-8

12. Wadhwa S, Kapila S. TMJ disorders: future innovations in diagnostics and therapeutics. J Dent Educ 2008;72(8):930-47

13. Huang G], LeResche L, Critchlow CW, Martin MD, Drangsholt MT. Risk factors for diagnostic subgroups of painful temporomandibular disorders (TMD). J Dent Res 2002;8I (4):284-8

14. Buescher J). Temporomandibular joint disorders. Am Fam Physician 2007;76( I0): 1477-82

15. Slade GD, Fillingim RB, Sanders AE, et al. Summary of findings from the OPPERA prospective cohort study of incidence of first-onset temporomandibular disorder: implications and future directions. J Pain 2013; | 4(I2 Suppl):T| | 6-24

16. Kast RE. Tiagabine may reduce bruxism and associated temporomandibular joint pain. Anesth Prog 2005;52(3): 102-4
17. Hasanain F, Durham J, Moufti A, Steen IN, Wassell RW. Adapting the diagnostic definitions of the RDC/TMD to routine clinical practice: a feasibility study. J Dent 2009;37( I 2):955-62

18. International RDC-TMD Consortium. DC/TMD. 2014. Available from: http://www.rdc-tmdinternational.org/ Home.aspx (Accessed 29 April 2014)

19. Gray RJ, Al-Ani Z. Conservative temporomandibular disorder management: what DO I do? - frequently asked questions. Dent Update 20 | 3;40(9):745-8,5 I-2,54-6

20. Stacey D, Legare F, Col NF, et al. Decision aids for people facing health treatment or screening decisions. Cochrane Database Syst Rev 20 14; 1:CD00 | 43 |

21. Staff H. Temporomandibular disorder: should I have surgery for jaw pain? 2012. Available from: https://www.healthwise.net/cochranedecisionaid/Content/ StdDocument.aspx?DOCHWID=tn8353 (Accessed 2 May 2014)

22. Sarah C. 'What matters most to you?' How decision aids help patients make better choices. 2014. Available from: http://www.evidentlycochrane.net/what-matters-most-toyou-how-decision-aids-help-patients-make-better-choices2/ (Accessed 2 May 2014)

23. Navratil L, Navratil V, Hajkova S, Hlinakova P, Dostalova T, Vranova J. Comprehensive treatment of temporomandibular joint disorders. Cranio 2014;32(I):24-30

24. Lin J, Zhang W, Jones A, Doherty M. Efficacy of topical non-steroidal anti-inflammatory drugs in the treatment of osteoarthritis: meta-analysis of randomised controlled trials. Br Med J 2004;329(746 I):324. Epub 2004/08/03

25. Mujakperuo HR, Watson M, Morrison R, Macfarlane TV. Pharmacological interventions for pain in patients with temporomandibular disorders. Cochrane Database Syst Rev 2010(10):CD0047/5. Epub 2010/10/12

26. Blenkiron DP. Cognitive Behavioural Therapy. 2013. Available from: http://www.rcpsych.ac.uk/mentalhealthinformation/therapies /cognitivebehaviouraltherapy.aspx (Accessed I May 20|4)

27. Litt MD, Porto FB. Determinants of pain treatment response and nonresponse: identification of TMD patient subgroups. J Pain 2013; |4(| |):1502-13

28. Liu HX, Liang QJ, Xiao P, Jiao HX, Gao Y, Ahmetjiang A. The effectiveness of cognitive-behavioural therapy for temporomandibular disorders: a systematic review. J Oral Rehabi. 2012;39( I):55-62

29. Calderon Pdos S, Tabaquim Mde L, Oliveira LC, Camargo AP, Ramos Netto Tde C, Conti PC. Effectiveness of cognitive-behavioral therapy and amitriptyline in patients with chronic temporomandibular disorders: a pilot study. Braz Dent J 201 I;22(5):4 I 5-21

30. Klasser GD, Greene CS. Oral appliances in the management of temporomandibular disorders. Oral Surg Oral Med Oral Pathol Oral Radiol Endod 2009; 107(2):212-23 


\section{APPENDIX}

\section{MORECAMBE BAY MAXILLOFACIAL SERVICES TEMPROMANDIBULAR (JAW) JOINT PROBLEMS}

\section{WHAT ARETHEY?}

The joint between your upper and lower jaws, just in front of your ears, is called the temporomandibular joint (TMJ for short). A disc (cartlidge) separates the bones in the upper and lower jaws. The jaw joints can occasionally cause problems, which your dentist can help diagnose.

\section{WHAT ARETHE SYMPTOMS?}

- Clicking or grating when you move your mouth

- Difficulty opening or closing your mouth

- Swelling or tenderness over the joint

- Pain in the face, neck and chewing muscles (some people also have pain in the shoulders and or back)

- Headaches or migraines, especially first thing in the morning

- Pain in your ear, possibly with ringing noises

Clicking and grating noises are usually down to the cartilage jumping when you open your mouth. The problem can be addressed by:

- Cutting up food into smaller pieces

- Avoiding hard or sticky or chewy foods

- Try not to open your mouth too wide - for example when yawning

Jaw joint pain is usually the result of increased tension in the jaw muscles.

Factors involved in this include:

- Grinding or clenching your teeth, in your sleep or during the day

- Habits such as biting your nails or chewing pens

These are often related to stress, fatigue and anxiety.

Painkillers and sedatives can relieve the symptoms in the short term, possible solutions to the underlying problems include:

- Using relaxation and stress relieving techniques

- Giving up habits that aggravate the condition

- Wearing a 'bite guard' at night, to stop you grinding your teeth in your sleep

- Jaw exercises

The purpose of the exercises is to prevent any clicking of the jaw joint and to strengthen the muscles which pull the jaw backwards. This in turn will relax the muscles.

Set aside two five-minute periods each day at a time when you are relaxed, sitting upright on a chair and carry out the following manoeuvers:

- Close your mouth on your back teeth, resting the tip of your tongue on your palate just behind the upper front teeth

- Run the tip of your tongue backwards onto the soft palate as far back as it will go, keeping your teeth together

- Force the tongue back to maintain contact with the soft palate and slowly open your mouth until you feel your tongue just being pulled away from it, do not try to open your mouth further, relax for five seconds

- Repeat this manoeuvre slowly over the next five minutes in a firm but relaxed fashion

As you open your mouth you should feel tension in the muscles at the back of your jaw beneath your chin. Do this exercise no more than the recommended amount for the first week, thereafter do this as often as you can. 